\title{
Kernos
}

Revue internationale et pluridisciplinaire de religion grecque antique

$25 \mid 2012$

Varia

\section{Daniela GIACOMETTI, Metaponto. Gli dei e gli eroi nella storia di una polis di Magna Grecia}

\author{
Ilaria Battiloro
}

\section{OpenEdition}

\section{Journals}

Edizione digitale

URL: http://journals.openedition.org/kernos/2069

DOI: 10.4000/kernos.2069

ISSN: 2034-7871

\section{Editore}

Centre international d'étude de la religion grecque antique

\section{Edizione cartacea}

Data di pubblicazione: 26 ottobre 2012

Paginazione: $365-367$

ISSN: 0776-3824

Notizia bibliografica digitale

Ilaria Battiloro, « Daniela GIACOMETTı, Metaponto. Gli dei e gli eroi nella storia di una polis di Magna

Grecia », Kernos [En ligne], 25 | 2012, mis en ligne le 01 octobre 2012, consulté le 21 septembre 2020

URL : http://journals.openedition.org/kernos/2069 ; DOI : https://doi.org/10.4000/kernos.2069

Questo documento è stato generato automaticamente il 21 settembre 2020.

Kernos 


\title{
Daniela GIACOMETTI, Metaponto. Gli dei e gli eroi nella storia di una polis di Magna Grecia
}

\author{
Ilaria Battiloro
}

\section{NOTIZIA}

Daniela GIACOMETTI, Metaponto. Gli dei e gli eroi nella storia di una polis di Magna Grecia, Cosenza, Edizioni Lionello Giordano, 2005. 1 vol. $15 \times 21,5 \mathrm{~cm}, 271$ p. (Biblioteca di Studi Religiosi, 6. Studi e Testi, 1). ISBN : 88-86919-22-0.

Come sottolinea G. Casadio nella postfazione al volume di D. Giacometti (p. 239-265), l'interesse per gli aspetti cultuali del mondo greco accomuna una serie di recenti studi regionali tra cui - nell'ambito delle ricerche sulle poleis greche d'Occidente - si segnala il volume dedicato ai culti di Taranto pubblicato da E. Lippolis, S. Garraffo e M. Nafissi (Culti greci in Occidente. Fonti scritte e documentazione archeologica, I. Taranto, Taranto, 1995).

2 Il tema di fondo dello studio della Giacometti (D.G.) è la ricostruzione delle vicende storiche e politiche della colonia achea di Metaponto e il suo sviluppo urbano dalla fondazione fino al IV sec. a.C., attraverso l'analisi dei culti e delle divinità venerate, una scelta quasi "obbligata", questa, in un ambito, quale quello greco-coloniale, in cui i momenti fondamentali di una polis sono segnati da atti rituali (p.11). Passando ad analizzare da vicino il contenuto del libro, la materia è suddivisa in tre grandi sezioni.

3 La prima parte («Gli dei», p. 15-125) offre una rassegna delle divinità la cui presenza è attestata a Metaponto da fonti scritte e archeologiche; la seconda sezione («Gli eroi», p. 127-183) è invece dedicata agli eroi della città, mentre la terza ed ultima sezione del volume («La polis e i santuari panellenici», p. 185-202) analizza i rapporti tra la colonia achea e i santuari panellenici. Nelle due sezioni dedicate a dei ed eroi, l'A. procede in ordine alfabetico. Per ogni figura, vengono censite e riportate prima le fonti scritte, con 
traduzione dell'A. stessa, poi si riportano i dati delle evidenze archeologiche relative alla divinità od eroe analizzato. Dopo aver censito i dati, D.G. discute criticamente le evidenze relative a ciascuna divinità e culto. La medesima procedura viene utilizzata nell'esame delle relazioni intrattenute da Metaponto con i santuari panellenici della Grecia peninsulare (Delfi, Delo e Olimpia). I culti così analizzati rappresentano la base per la ricostruzione storica delle vicende metapontine dalla fondazione al IV sec. a.C., cui è dedicata la parte conclusiva del volume («Conclusioni», p. 203-218). Il quadro che di Metaponto emerge dal lavoro di ricostruzione storico-religiosa proposto da D.G. è di una polis la cui identità religiosa muta e si trasforma con l'evolversi delle vicende storico-politiche che la coinvolgono.

Nella sua prima fase di vita successiva alla fondazione, la città achea definisce la sua identità religiosa intorno a tre divinità femminili, garanti dell'ordine della comunità metapontina: Hera, divinità poliade; Artemis, che secondo le fonti accompagnò i coloni "nella città nutrice di cavalli", e la "dolce" Afrodite (p. 206 sg.). Alle prime due dee sono, infatti, dedicati i santuari più importanti dell'area metapontina, il santuario di San Biagio e il cd. santuario delle Tavole Palatine. A questi due importanti santuari extraurbani volti a "delimitare" il territorio metapontino nella sua fase di definizione si aggiungono $i$ due più antichi templi del santuario urbano. Il tempio $C$, infatti, tradizionalmente attribuito al culto di Athena, è invece considerato un Artemision da D.G. L'attribuzione è corroborata da fattori di natura archeologica: i materiali provenienti dal santuario extraurbano di San Biagio presentano forti analogie con quelli rinvenuti dall'area intorno alla peristasis del tempio C, il cui sacello C I costituisce la più antica costruzione templare del complesso urbano. L'ipotesi di un culto di Artemis, tra l'altro già ventilata dalla Dilthey ${ }^{1}$, è dunque considerata più plausibile della communis opinio che vuole invece il tempio $\mathrm{C}$ dedicato ad Athena, sulla base del rinvenimento di un cippo dedicato ad Athena Hygieia lungo la linea di temenos adiacente all'oikos (p. 67). A ciò si aggiunge l'analogia tra il fregio fittile con corte di tre donne rinvenuto nell'area della peristasis del tempio $\mathrm{C}$ con un fregio analogo proveniente da San Biagio: si tratta della raffigurazione del rituale che introduce al matrimonio, in cui le due figure femminili sul carro sono interpretabili come la sacerdotessa della dea e la nymphe per eccellenza, ovvero Kore, mentre le tre donne che precedono il carro sono interpretate come le Proitides nelle vesti delle Charites (le Proitides sono legate ad Artemis in una delle due versioni del mito che le vede protagoniste, in cui, fatte impazzire da Hera, sono successivamente guarite da Artemis). Il legame con la sfera matrimoniale accomunerebbe, quindi, il culto di Artemis praticato a san Biagio con il culto della stessa dea nel santuario urbano (oikos C). Il quadro che scaturisce dall'attribuzione del tempio $\mathrm{C}$ ad Artemis è quello di «una realtà cultuale in cui alla dedica delle due dee, Hera e Artemis, dei due più importanti santuari della chora (quello delle Tavole Palatine e quello di S. Biagio) farebbe da pendant la dedica di due dei più antichi templi del santuario urbano: più $o$ meno contemporaneamente alla costruzione del sacello C (l'Artemision) e della fase più antica di D (l'Aphrodision), orientati secondo l'orientamento sacro', vengono poste le fondamenta del tempio A I (l'Heraion, mai realizzato in questa prima fase), il cui orientamento è solo leggermente sfalsato rispetto a quello di C» (cit. p. 70-71). Ad Aphrodite, infine, è dedicato il tempio ionico $\mathrm{D}$, ubicato nella zona settentrionale del santuario urbano. Monumentalizzato all'inizio del V sec. a.C., il tempio D conserva, insieme all'oikos C, il vecchio 'orientamento sacro', fattore che sembrerebbe riflettere un legame tra Artemis e Aphrodite (p. 27). 
5 A metà del VI sec. a.C., successivamente alla distruzione di Siris, la storia religiosa e politica di Metaponto è segnata da un cambiamento. Se la divinità poliade rimane Hera, «l'identità della comunità dei politai di Metaponto si ridefinisce intorno alla coppia Hera-Apollon Lykeios», ed è a loro che vengono dedicati i due grandi templi AII (Heraion) e BII (Apollonion) costruiti nell'ultimo trentennio del VI sec. Questo volgersi verso Apollo, e non più verso la sorella Artemis, riflette, come si è detto, una mutata situazione politica, ovvero l'esperienza tirannica metapontina cui è legato il fenomeno dell'emergere dell'oplitismo. Apollon Lykeios, infatti, è il dio degli efebi armati, dei cittadini-soldati, ai quali pare debba essere attribuita la dedica degli argoi e tetragonoi lithoi, nonché dei cippi e stelai rinvenuti nell'area del santuario (p. 209). All'inizio del V sec. si data la monumentalizzazione dell'area settentrionale del santuario e del tempio ionico D (l'Aphrodision), in concomitanza con la monumentalizzazione dell'oikos arcaico C. Entrambi gli edifici conservano, successivamente a questa monumentalizzazione, l'originario 'orientamento sacro', fattore che denuncia un ritorno al linguaggio artistico dell'epoca della fondazione della città. Contemporanea è anche la costruzione del bomos in onore di Apollon eretto nell'agora su ordine di Aristea di Proconneso, come risultato dello stretto legame intessuto da Pitagora, Aristea e il metapontino Parmenisco con il santuario di Apollon a Delos.

Degli altri edifici contemporanei, gli oikoi $\mathrm{F}$ e $\mathrm{G}$ ubicati a sud del tempio $\mathrm{C}$, uno dei due era certamente dedicato ad Athena, venerata come Hygieia, epiclesi con la quale la dea, prima che a Metaponto, è venerata solo ad Atene, probabile riflesso di un nuovo legame tra Metaponto e la polis attica sancito, tra l'altro, da un xymmachikon risalente al 433 a.C. In conseguenza della sconfitta ateniese in Sicilia, anche le sorti di Metaponto cominciano a vacillare, fino a quando, nel corso del IV sec. a.C., la colonia achea comincia a gravitare nell'orbita tarantina, pur non perdendo la propria indipendenza, come testimoniano le vicende tarantine e metapontine della fine del secolo.

Quando si termina la lettura del nucleo interpretativo del libro, rappresentato dalle "Conclusioni», ci si rende conto che l'A. ha raggiunto lo scopo prefissato ed esplicitamente dichiarato nell'introduzione, ovvero di «ricostruire la storia di una polis achea della Magna Grecia attraverso i suoi culti» (p.11). Oltre che per alcune aggiornate riflessioni su problematiche di carattere storico proposte dall'A. (tra cui si segnala la complessa vicenda delle Proitides e il legame di questo mito con Hera e Artemis [p. 53-75], nonché la questione del culto di Apollon Lykeios, attestato in ambito magno-greco solo a Metaponto e connesso alla difficile interpretazione degli argoi e tetragonoi lithoi [p. 39-42]), va riconosciuto all'A. il merito di aver raccolto e discusso tutte le fonti, letterarie, epigrafiche numismatiche ed archeologiche, inerenti la vita religiosa della colonia achea dalla fondazione al IV sec. a.C. Il volume, quindi, è certamente uno strumento irrinunciabile per quanti vogliano accostarsi alle principali tematiche inerenti la storia politico-religiosa di Metaponto e seguirne l'evoluzione nella diacronia. 


\section{NOTE}

1. H. DILTHEY, «Sorgenti acque luoghi sacri in Basilicata», in Attività archeologica in Basilicata (1964-1977). Scritti in onore di D. Adamesteanu, Matera, 1980, p. 548-550.

\section{AUTORI}

\section{ILARIA BATTILORO}

Mt. Allison University 